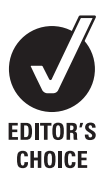

'Dermatology Department, Complexo Hospitalario de Pontevedra, Pontevedra, Spain ${ }^{2}$ Research Unit, Sociedad Española de Reumatología, Madrid, Spain

${ }^{3}$ Clinical Research Unit,

Complejo Hospitalario

Universitario de Albacete, Albacete, Spain

${ }^{4}$ Hospital San Jorge, Huesca, Spain

${ }^{5}$ Hospital Clínic i Provincial, Barcelona, Spain

${ }^{6}$ Rheumatology Service and Department of Medicine,

Hospital Clínico Universitario, Medical School, Universidad de Santiago de Compostela, Santiago, Spain

\section{Correspondence to} Loreto Carmona, Research Unit, Sociedad Española de Reumatología, Calle Marqués del Duero, 5, 28001 Madrid, Spain;

loreto.carmona@ser.es

Accepted 12 May 2010

\title{
Incidence and risk of hospitalisation due to shingles and chickenpox in patients with rheumatic diseases treated with TNF antagonists
}

\author{
Ignacio García-Doval, ${ }^{1}$ Beatriz Pérez-Zafrilla, ${ }^{2,3}$ Miguel Ángel Descalzo, ${ }^{2}$ Rosa Roselló, ${ }^{4}$ \\ $M^{a}$ Victoria Hernández, ${ }^{5}$ Juan J Gómez-Reino, ${ }^{6}$ Loreto Carmona ${ }^{2}$; BIOBADASER 2.0 \\ Study Group
}

\begin{abstract}
Objective To estimate the incidence of hospitalisation due to varicella zoster virus (VZV) infection in patients treated with tumour necrosis factor (TNF) antagonists for inflammatory rheumatic conditions and to compare it with the expected rate in the general population.

Methods Secondary data analysis was performed of two large databases: (1) the national registry of rheumatic diseases patients treated with biological agents (BIOBADASER); and (2) the national hospital discharge database Conjunto Mínimo Básico de Datos al Alta Hospitalaria. Hospitalisations due to shingles or chickenpox were analysed. For each condition the incidence rate (IR) and the age and gender standardised IR per 100000 person-years plus the standardised incidence ratio (SIR) and the standardised incidence difference (SID) were estimated.
\end{abstract}

Results In patients exposed to TNF antagonists, the estimated IR of hospitalisation due to shingles was 32 cases per 100000 patient-years (95\% Cl 14 to 78 ), the expected rate in the general population was 3.4 (95\% Cl 3.2 to 3.5), the SIR was 9 (95\% Cl 3 to 20 ) and the SID was 26 (95\% Cl 14 to 37). The estimated IR of hospitalisation due to chickenpox was 26 cases per 100000 (95\% Cl 10 to 69), the expected rate was 1.9 (95\% Cl 1.8 to 2.0), the SIR was 19 (95\% Cl 5 to 47$)$ and the SID 33 (95\% Cl 21 to 45).

Conclusions Patients suffering rheumatic diseases exposed to TNF antagonists are hospitalised due to VZV infections significantly more frequently than expected in the general population. Since the absolute IR of hospitalisations due to chickenpox and shingles is low in these patients, the implementation of risky preventive measures may not be justified at present.

\section{INTRODUCTION}

Primary infection by the varicella zoster virus (VZV) causes chickenpox, commonly in children or young adults. Herpes zoster or shingles is caused by the reactivation of latent VZV infection in association with a decline in VZV-specific cell-mediated immunity. The world annual incidence rate (IR) of shingles ranges from 1.2 to 3.4 cases per 1000 healthy individuals, increasing to 3.9-11.8 per year per 1000 individuals aged $>65$ years or immunocompromised. ${ }^{12}$ Both chickenpox and shingles may have devastating consequences in immunocompromised hosts. ${ }^{1}$

Tumour necrosis factor (TNF) antagonists are increasingly used for the treatment of immune-mediated inflammatory diseases including rheumatic disorders. Treatment with TNF antagonists is associated with an increased risk of tuberculosis ${ }^{3}$ and of opportunistic infections in general. However, the extent to which this risk is related to the use of TNF antagonists or to the disease itself remains a subject of debate. The association between TNF antagonists and viral infections needs further clarification. There is a biological basis for an increased risk of these infections in patients treated with TNF antagonists. ${ }^{4}$ Two analyses of large databases concerning the association of TNF antagonists and VZV infections in patients with rheumatic disease ${ }^{5}$ show conflicting results. An increased rate of herpes zoster associated with monoclonal anti-TNF antibodies (adalimumab and infliximab) was recently reported in patients with rheumatoid arthritis (RA). ${ }^{7}$ The clinical relevance of the increase was uncertain.

In patients treated with TNF antagonists, prevention of severe VZV infection leading to hospital admission by vaccination or otherwise may have a favourable benefit/RR if rates are clinically relevant. Previous strategies of screening and treating latent tuberculosis infection in patients prior to treatment with TNF antagonists have been successful. ${ }^{8}$ The objective of the present analysis is to evaluate the magnitude of the problem of VZV infections leading to hospital admission in patients with rheumatic disease treated with TNF antagonists.

\section{PATIENTS AND METHODS}

The incidence of hospitalisation due to VZV infections was estimated from two sources: (1) a national active registry of patients with rheumatic diseases treated with TNF antagonists (Registro Español de Acontecimientos Adversos de Terapias Biológicas en Enfermedades Reumaticas; BIOBADASER) and (2) an administrative database of all hospital admissions in public centres in Spain (Conjunto Mínimo Básico de Datos al Alta Hospitalaria; CMBD). Only patients aged $\geq 18$ years were analysed.

Spain has universal health coverage, yet $17 \%$ of the population uses private healthcare resources. TNF antagonists are provided at no cost in public hospitals. The Spanish population has a constrictive pyramid age distribution and a life expectancy (2005-2010) at birth of 84 years for women and 78 years for men (United Nations Statistics Division). 


\section{Patients exposed to TNF antagonists: the BIOBADASER registry}

The BIOBADASER registry has been described in detail previously, ${ }^{89}$ and the complete protocol is available at its website (http://biobadaser.ser.es). In brief, BIOBADASER is a registry established in February 2000 for the active long-term follow-up of the safety of biological therapies in patients with rheumatic diseases in Spain. The registry registers patients beginning treatment with biological therapies in the participating centres and includes all rheumatic diseases. Data are reported online by participating rheumatologists using electronic forms that are monitored on a weekly basis. Patient recruitment, modifications of treatment and adverse events are communicated at the time they occur. Once a year-or occasionally every 6 months in the case of recently approved drugs-data are validated by onsite audits. The second phase of BIOBADASER, known as BIOBADASER 2.0, was launched in July 2006. To improve data quality, this registry only includes data from 14 large public hospitals with more than 100 patients that participated in the first phase beginning in 2000. Additional requirements for inclusion in BIOBADASER 2.0 are written commitment by rheumatologists to collect the same data as in the first phase, and availability of all patients who gave informed consent to be contacted by telephone for further data validation (specifically for any hospitalisation in the last year). The BIOBADASER 2.0 protocol and materials were approved by the Institutional Review Board of the Hospital Ramón y Cajal, Madrid.

Data in BIOBADASER 2.0 include: (1) gender, date of birth, diagnosis, date of diagnosis, diagnosis specifications and comorbidities; (2) treatment data; and (3) data on adverse events, including definition according to the medical dictionary of regulatory activities, ${ }^{10}$ severity, outcome and concomitant nonantirheumatic medications. The main variable in BIOBADASER is the relevant adverse event, including both severe and nonsevere adverse events, related or unrelated to medications.

Only patients aged $\geq 18$ years and receiving TNF antagonists in BIOBADASER 2.0 were included in this study.

\section{Population data: CMBD}

The CMBD is an administrative database of all hospital discharge records in Spain, launched in 1987. It includes data for all admissions to hospitals belonging to or collaborating with the National Health System. Gender, age at admission and main diagnosis coded using ICD-9-CM are recorded. Private hospitals not included in this database represent $17.1 \%$ of all admissions according to the National Statistics Institute. ${ }^{11}$ For the present analysis we used data from 1 January 2003 to 31 December 2006 for all cases with chickenpox (052.X) or herpes zoster infection (053.XX) as the main diagnosis and age $\geq 18$ years.

\section{Case definition}

Cases were defined as an infection by VZV (chickenpox or shingles) which was reported to BIOBADASER and the infection had resulted in a hospital admission.

\section{Statistical analysis}

We estimated the IR of hospitalisation due to VZV infection per 100000 patient-years with 95\% CI. Time of exposure in BIOBADASER 2.0 was the period from the starting date of the first TNF antagonist until discontinuation, death, loss to follow-up, hospitalisation due to VZV infection or end of follow-up (1 September 2008). If a patient had a TNF antagonist discontinued and then restarted during these two time points, the exposure time was the sum of the exposure times for each TNF antagonist. The IR in the general population $\mathrm{CMBD}$ was calculated using a denominator of 28.6 million for each year of the study period. This denominator represents the $83 \%$ of the Spanish population aged $\geq 18$ years covered by the National Health System according to data from the National Statistics Institute, ${ }^{12}$ excluding the population covered by private hospitals $(17.1 \%)$. We also estimated the age- and gender-standardised IR by the indirect method. The reference population for age and gender adjustments was obtained from the National Statistics Institute, which provided figures for the Spanish population in 2003-6.12 Based on the standardised IR estimated for observed (BIOBADASER) and expected (CMBD) cases, we calculated the standardised incidence ratio (SIR) and the standardised incidence difference (SID).

All analyses were performed using Stata V.10.0 (Stata, College Station, Texas, USA).

\section{RESULTS}

\section{Study population}

Figure 1 shows an overview of the databases analysed. BIOBADASER 2.0 yielded information on 4655 patients receiving TNF antagonists, with a total exposure of 15389 patientyears. The baseline characteristics of these patients are shown in table 1 . The most frequent diagnoses were RA ( $n=2562,55 \%)$, ankylosing spondylitis $(\mathrm{n}=759,16 \%)$ and psoriatic arthritis $(n=723,15 \%)$. A total of 1250 patients were treated with more than one TNF antagonist. Infliximab was used in 2618 (41\%) treatments, etanercept in 2054 (32\%) and adalimumab in 1436 $(23 \%)$.

\section{Hospitalisations due to shingles}

From 2003 to 2006, 3825 discharges with the diagnosis of shingles as cause of admission were recorded in the CMBD. Thus, the expected standardised IR of hospitalisation due to shingles per 100000 person-years was 3.4 (95\% CI 3.2 to 3.5) (table 2). The rate was significantly higher in men (3.6 (95\% CI 3.5 to 3.8)) than in women (3.1 (95\% CI 3.0 to 3.2$)$ ).

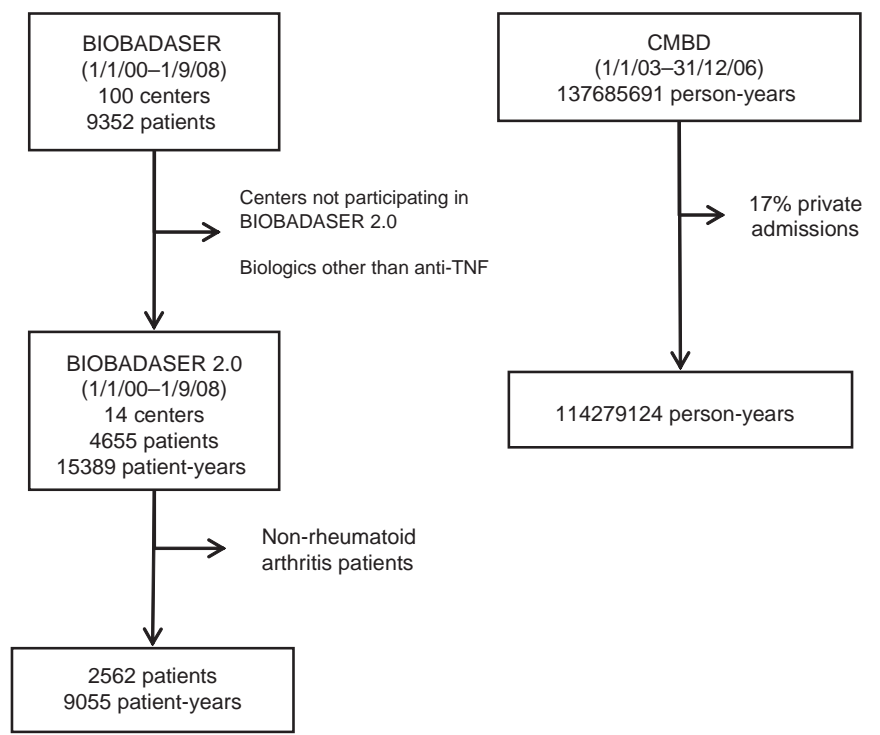

Figure 1 Databases and patients included in the study analyses. BIOBADASER, Registro Español de Acontecimientos Adversos de Terapias Biológicas en Enfermedades Reumaticas; CMBD, Conjunto Mínimo Básico de Datos al Alta Hospitalaria. 
In BIOBADASER 2.0 there were 106 cases of shingles reported (IR 65.6 per 10000 patient-years; 95\% CI 54 to 79.8), 5 of which resulted in hospitalisation. All cases occurred during anti-TNF therapy. The estimated IR of hospitalisations for shingles in exposed patients was 32 cases per 100000 patient-years (95\% CI 14 to 78), and 29 (95\% CI 9 to 67) after adjustment for age and gender (table 2). The SIR and SID for hospitalisation due to shingles infection in patients treated with TNF antagonists compared with the general population were 9 (95\% CI 3 to 20) and 26 (95\% CI 14 to 37) per 100000 patient-years, respectively. No fatalities occurred.

In patients with RA exposed to TNF antagonists in BIOBADASER 2.0, the IR per 100000 patient-years was 44 (95\% CI 17 to 118) and the age- and gender-adjusted IR was 34 (95\% CI 9 to 87 ) (table 2).

\section{Hospitalisation due to chickenpox}

In the CMBD there were 2163 discharges (aged $\geq 18$ years) for chickenpox. The background IR of hospitalisation due to chickenpox was 1.9 cases per 100000 person-years (95\% CI 1.8 to 2.0) (table 3). The IR in men (2.6 (95\% CI 2.4 to 2.7$)$ ) was significantly higher than in women (1.3 (95\% CI 1.2 to 1.4$))$.

In BIOBADASER there were 13 cases of chickenpox, 4 of whom met the case definition and were hospitalised (table 3), so the estimated IR of hospitalisation due to chickenpox in patients

Table 1 Baseline characteristics of patients with rheumatic diseases exposed to TNF antagonists

\begin{tabular}{lcc}
\hline & All diseases & Rheumatoid arthritis \\
\hline $\mathrm{N}$ & 4655 & 2562 \\
Women (n (\%)) & $2910(63)$ & $2045(80)$ \\
Mean (SD) age (years) & $50(14)$ & $54(13)$ \\
Mean (SD) disease duration (years) & $11(9)$ & $10(8)$ \\
Rheumatoid factor positive (n (\%)) & & $2341(91)$ \\
Mean (SD) DAS28 & & $5.5(1.3)$ \\
Methotrexate use (n (\%)) & $2078(45)$ & $1282(50)$ \\
Other DMARD use (n (\%)) & $463(10)$ & $190(7)$ \\
Corticosteroid use (n (\%)) & $1770(38)$ & $1240(48)$ \\
\hline
\end{tabular}

DAS28, disease activity score; DMARD, disease-modifying antirheumatic drug; TNF, tumour necrosis factor. with rheumatic disease treated with a TNF antagonist was 26 cases per 100000 (95\% CI 10 to 69). After adjusting for age and gender, the rate was 35 (95\% CI 9 to 91) per 100 000. The SIR in patients treated with a TNF antagonist was 19 (95\% CI 5 to 47) and the SID was 33 cases per 100000 patient-years (95\% CI 21 to 45). No fatal cases occurred. No cases occurred in patients with RA exposed to TNF antagonists.

Selected characteristics of the cases of VZV infection in BIOBADASER 2.0 who were hospitalisation are shown in table 4. All four cases of chickenpox occurred in young adults in or around the first year of treatment.

\section{DISCUSSION}

In this study we found that patients with rheumatic diseases exposed to TNF antagonists had a 10-fold increase in the rate of hospitalisation due to VZV infections compared with the general population. For completeness, we also calculated the risk in the subset of patients with RA, who are characterised by a high rate of shingles. ${ }^{5}$ Unfortunately, the analysis presented here does not allow differentiation between the main causes for this increased risk (treatment with TNF antagonists, the underlying diseases or other medications such as steroids). On the other hand, our results show that the absolute rate of hospitalisations due to chickenpox and shingles in exposed patients is low (about 3 cases per 10000 person-years of exposure for shingles and for chickenpox).

We have maintained a control cohort of RA patients for 5 years (Estudio de la Morbilidad y Expresión Clínica de la Artritis Reumatoide; EMECAR), which has been used in previous studies to compare outcomes in TNF-exposed and non-exposed patients with RA. ${ }^{1314}$ We have previously reported that the rate of shingles of any severity in patients with RA in BIOBADASER was not statistically different from EMECAR (IRR $2.7(95 \%$ CI 0.7 to 22.9$)$ ). ${ }^{1516}$ However, the measurement of non-hospitalised cases of infection is somewhat different between the two cohorts, so we decided not to present those data here. The point estimate (2.7), however, does not differ much from published reports on the risk of herpes infection in patients exposed to

Table 2 Incidence of hospitalisations due to shingles in population aged $\geq 18$ years exposed to TNF antagonists

\begin{tabular}{|c|c|c|c|c|c|}
\hline & Cases & Person-years & $\begin{array}{l}\text { Incidence rate per } \\
100000(95 \% \mathrm{Cl})\end{array}$ & $\begin{array}{l}\text { SIR per } 100000 \\
(95 \% \mathrm{Cl})\end{array}$ & SIR (95\% CI) \\
\hline \multicolumn{6}{|l|}{ BIOBADASER 2.0} \\
\hline $\begin{array}{l}\text { All rheumatic diseases exposed to TNF } \\
\text { antagonist }\end{array}$ & 5 & 15389 & 32 (14 to 78 ) & 29 (9 to 67$)$ & 9 (3 to 20) \\
\hline $\begin{array}{l}\text { Rheumatoid arthritis exposed to TNF } \\
\text { antagonist }\end{array}$ & 4 & 9055 & 44 (17 to 118$)$ & 34 (9 to 87 ) & 10 (3 to 26$)$ \\
\hline CMBD (expected) & 3825 & 114279124 & 3.4 (3.2 to 3.5$)$ & $3.4(3.2$ to 3.5$)$ & \\
\hline
\end{tabular}

BIOBADASER, Registro Español de Acontecimientos Adversos de Terapias Biológicas en Enfermedades Reumáticas; CMBD, Conjunto Mínimo Básico de Datos al Alta Hospitalaria (Hospital Discharge Database); SIR, standardised incidence ratio; TNF, tumour necrosis factor.

Table 3 Incidence of hospitalisations due to chickenpox in population aged 18 years exposed and not exposed to TNF antagonists

\begin{tabular}{|c|c|c|c|c|c|}
\hline & Cases & Person-years & $\begin{array}{l}\text { Incidence rate per } \\
100000(95 \% \mathrm{CI})\end{array}$ & $\begin{array}{l}\text { SIR per } 100000 \\
(95 \% \mathrm{CI})\end{array}$ & SIR (95\% CI) \\
\hline \multicolumn{6}{|l|}{ BIOBADASER 2.0} \\
\hline $\begin{array}{l}\text { All rheumatic diseases exposed to TNF } \\
\text { antagonist }\end{array}$ & 4 & 15395 & 26 (10 to 69$)$ & 35 (9 to 91$)$ & $19(5$ to 47$)$ \\
\hline $\begin{array}{l}\text { Rheumatoid arthritis exposed to TNF } \\
\text { antagonist }\end{array}$ & 0 & 9066 & & & \\
\hline CMBD (expected) & 2163 & 114279124 & $1.9(1.8$ to 2.0$)$ & $1.9(1.8$ to 2.0$)$ & \\
\hline
\end{tabular}

BIOBADASER, Registro Español de Acontecimientos Adversos de Terapias Biológicas en Enfermedades Reumáticas; CMBD, Conjunto Mínimo Básico de Datos al Alta Hospitalaria (Hospital Discharge Database); SIR, standardised incidence ratio; TNF, tumour necrosis factor. 
Table 4 Characteristics of cases with hospitalisation due to varicella zoster virus infection in BIOBADASER 2.0

\begin{tabular}{|c|c|c|c|c|c|c|c|c|c|}
\hline Gender/Age & Diagnosis & $\begin{array}{l}\text { Disease duration } \\
\text { (years) }\end{array}$ & Treatment & $\begin{array}{l}\text { Treatment } \\
\text { duration (years) }\end{array}$ & $\begin{array}{l}\text { Treatment } \\
\text { discontinuation }\end{array}$ & MTX & Steroids & Infection & Extension \\
\hline $\mathrm{M} / 32$ & AS & - & Infliximab & 0.41 & Yes & Yes & No & Chickenpox & Pneumonia \\
\hline $\mathrm{M} / 32$ & AS & 8.1 & Etanercept & 0.99 & Yes & No & No & Chickenpox & Skin only \\
\hline$F / 34$ & PsA & 6.2 & Etanercept & 1.32 & Yes & No & Yes & Chickenpox & $\begin{array}{l}\text { Otitis with tympanic } \\
\text { membrane perforation }\end{array}$ \\
\hline $\mathrm{F} / 37$ & SCP & 1.9 & Infliximab & 1.01 & Yes & Yes & Yes & Chickenpox & Pneumonia \\
\hline $\mathrm{F} / 52$ & RA & 7.2 & Adalimumab & 2.11 & No & No & Yes & Shingles & Localised \\
\hline $\mathrm{F} / 56$ & AS & 24.8 & Infliximab & 2.77 & No & No & No & Shingles & Localised \\
\hline $\mathrm{F} / 61$ & RA & 6.3 & Etanercept & 5.66 & Yes & Yes & No & Shingles & Localised \\
\hline $\mathrm{M} / 73$ & RA & 6.4 & Infliximab & 0.69 & No & Yes & Yes & Shingles & Localised \\
\hline $\mathrm{F} / 74$ & RA & 3.2 & Etanercept & 0.76 & No & No & Yes & Shingles & Localised \\
\hline
\end{tabular}

AS, ankylosing spondylitis; BIOBADASER, Registro Español de Acontecimientos Adversos de Terapias Biológicas en Enfermedades Reumáticas; MTX, methotrexate; PsA, psoriatic arthritis; RA, rheumatoid arthritis; SCP, seronegative chronic polyarthritis.

anti-TNF agents. Since no VZV infections leading to hospitalisation occurred in the non-exposed EMECAR cohort, we were not able statistically to compare the rates between groups. We believe that the data presented here on hospitalisations due to VZV infection in patients with RA are not affected by reporting bias to either EMECAR or BIOBADASER. VZV infection in adults is a remarkable condition which is likely to be reported. In addition, the majority of participating rheumatologists contribute to both cohorts, so consideration of relevant and other medical decisions are performed by the same staff. Nevertheless, if we assigned one fictitious case of hospitalisation by shingles to our EMECAR cohort ( $n=789$, patient-years=2162), there would be no clear difference between the IR in the exposed and nonexposed patients (44 vs 46). Taking this into account, it is probable that the rate of shingles may not be increased in patients with RA exposed to TNF antagonists compared with those not exposed or, if there is an increase, it will not be large in absolute terms.

The CMBD is an administrative database which is not for claims purposes, so it is not exposed to several of the recognised sources of bias; claim databases may contain missing or inaccurate diagnostic codes because physician reimbursement schemes depend on medical action rather than on diagnosis. ${ }^{17}$ Nevertheless, data collection in the CMBD is not conducted for research purposes and case sensitivity needs to be considered. To avoid additional bias in comparing the rates in two different databases, we conducted a sensitivity analysis. The last annual audit in BIOBADASER showed that $7.4 \%$ of hospitalisations may not be reported. Applying this error to our data, with 5 hospitalisations due to shingles the rate could be between 6 cases (IR 39 per 100000 ) and 4 cases (IR 26 per 100 000). Additionally, the reliability of the information contained in the CMBD main diagnosis is $60 \%{ }^{18}$ so, assuming a $\pm 40 \%$ error of the 3825 cases, the rate would vary between 4.7 per 100000 and 2.0 per 100000 . In both cases the results do not substantially change our conclusions. Furthermore, in 1987, applying population-based estimates of chickenpox hospitalisations, Wharton ${ }^{19}$ estimated that 9300 hospitalisations due to chickenpox occur annually in the USA, although almost half of these were persons under 5 years of age. In another report, the rate of hospital admissions for chickenpox was in the range of 2.5 cases per 100000 adults, ${ }^{20}$ and in a third study the rate was 1.3.21 The background rates of hospitalisation for VZV infections in our report are therefore within the range of data reported by others, and our results are unlikely to have been significantly affected by the aforementioned limitations.

The main strength of our paper is the information on the rate of hospitalised cases of shingles and chickenpox in patients with rheumatic disease treated with TNF antagonists, which reflects the risk of potentially fatal or complicated infections and not just infections of any severity. Previous studies focused on herpes zoster infection and have shown that patients with RA have an increased risk, ${ }^{5-7}$ with rates of around 150 cases per 10000 person-years. Results on the additive effect of TNF antagonists in RA are conflicting. Smitten et at found an increased risk whereas Wolfe et al ${ }^{6}$ did not. Strangfeld et al estimated a crude IR of herpes zoster of 11.1 with monoclonal anti-TNF antibodies (adalimumab and infliximab), 8.9 with etanercept and 5.6 in controls. Our study was not powered to detect an additive effect of RA and TNF antagonists, but it shows a low level increase in the absolute risk of hospitalisations due to VZV disease associated with this exposure. In addition, we show evidence to suggest that between 1 and 7 cases of chickenpox and between 1 and 8 cases of shingles may be expected to need hospitalisation with exposure to TNF antagonists per 10000 rheumatic disease patient-years (see IR, CI in tables 2 and 3).

The main limitation of our study is that the case definition is based on hospitalisation, and hospitalisation may be more frequent in patients with some degree of immune suppression, as is the case in patients with RA. Moreover, Strangfeld et al showed a relation between risk and the need for biological therapy (propensity score), so the risk may be related more to an aggressive disease than to exposure to TNF antagonists. This additional effect could potentially lead to a fallacious increase in the rates of serious infection in exposed patients. Our finding of a 10-fold increase in the rate with respect to the rate in the background population could therefore be an additive effect of the increase in awareness in the immunosuppressed patient plus the fact that the VZV infection may be truly severe. As we consider the absolute increase in risk to be low, this effect would not invalidate our conclusion.

Experimental evidence supports the data reported in this study. Replication of VZV is inhibited in human fibroblast cells pretreated with recombinant TNF. This antiviral activity is completely blocked by the addition of monoclonal antibodies against TNF. Interleukin 12, interferon $\gamma$ and TNF also have an important role in the inhibition of the spread of VZV at an early stage of chickenpox. ${ }^{22}$ Overall, blocking TNF favours reactivation and spread of VZV and could lead to more severe disease.

Given the low overall risk, our study was underpowered to separate the effect of drugs and disease and to compare the risks associated with different drugs. Future research on TNF antagonists as a risk factor for severe VZV infection would require large cohorts and more specific questions that might only be feasible by aggregating data from existing registries.

It is very unlikely that our cohorts received systematic vaccination against VZV, as the general health mandate in Spain was given in 2005 and only for children aged 11-14 years. Standard guidelines for chickenpox vaccination probably apply to the population included in our study. ${ }^{23}$ Shingles vaccine is 
an attenuated vaccine with a higher dose of antigen that could potentially lead to more side effects in an immunosuppressed population. In a randomised clinical trial of adults aged $>60$ years, this vaccine was associated with 7 (95\% CI 1 to 13$)$ cases of severe adverse events and 14 (95\% CI 3 to 25) cases of vaccine-related systemic adverse events per 10000 vaccinations. ${ }^{24}$ The median duration of surveillance for herpes zoster was 3.1 years. These vaccination-associated risks are similar in rate and severity to the risks of hospitalised infections in our study. Hence, shingles vaccination before starting a TNF antagonist may not be warranted at present. In addition, it has been shown in Spain that the rate of severe cases (admissions) has not decreased with vaccination, ${ }^{25}$ so vaccination should clearly be recommended in healthy children but not in adults with immunosuppression secondary to the baseline inflammatory disease and its complications.

In conclusion, patients with rheumatic diseases exposed to TNF antagonists have a small absolute increase in the number of hospitalisations due to VZV infections. This modest increase suggests that the benefits of vaccination do not clearly outweigh the risks with regard to preventing serious or hospitalised herpes zoster infection.

The BIOBADASER 2.0 Study Group Agustí Sellas, Basilio Rodríguez y Mireia Barceló (Ciudad Sanitaria Vall d'Hebron); Laura Cebrián, María Montoro (Hospital Gregorio Marañon); Dolores Montesinos (Hospital Universitario Virgen Macarena); Eva Pérez-Pampín (Hospital Clinico Universitario de Santiago); Ana Mª Ortiz, (Hospital Universitario de La Princesa); Fred Antón, Antonio Zea (Hospital Ramon y Cajall; Francisco Javier Manero Ruiz, Chesús Beltrán, Eugenio Gimenez Úbeda, Fernando Jimenez Zorzo, Jesús Marzo, Marta Medrano, Ángela Pecondón (Hospital Universitario Miguel Servet); Mª Victoria Hernández, Raimon Sanmartí, Juan D Cañete (Hospital Clinic I Provincial); Carlos Rodriguez Lozano, Antonio Naranjo, Soledad Ojeda, Félix Francisco Hernández, Celia Erausquin, Íñigo Rúa, (Hospital de Gran Canaria Dr Negrin); Inmaculada Ureña, Maria Victoria Irigoyen, Laura Cano (Hospital General Carlos Haya); Rosa Roselló Pardo, Carlos Vazquez Galeano, (Hospital General San Jorge); Alexia de Juanes, Beatriz Joven, Javier Garcia (Hospital 12 de Octubre); Javier Calvo, Cristina Campos (Hospital General Universitario de Valencia); Juan Jose Garcia Borras, Rosa Negueroles, Luisa Muñoz, J L Valero, D Ybañez (Hospital La Fe).

Acknowledgements The authors gratefully acknowledge the Instituto de Información Sanitaria del Ministerio de Sanidad y Consumo, Spain for providing the CMBD data and Kathryn Fitch for editing the final manuscript.

Funding BIOBADASER is supported by the Spanish Society of Rheumatology and the Spanish Agency of Medicines and Healthcare Products. Grants in approximately equal amounts (all under $€ 25000 /$ year) from Roche, Abbott, BMS, Schering and Wyeth contribute to the support of the registry. This work was partially supported by RETICS Program, RD08/0075 (RIER) from Instituto de Salud Carlos III (ISCIII).

Competing interests IGD has no competing interest. MAD has no competing interest. BPZ has received lecture fees from Wyeth (€2000 total). RR has received honoraria from Abbott, Schering-Plough and Roche (€6000, €4000 and €1000/year, respectively). MVH has no competing interest. JJGR is on the Advisory Boards of Schering-Plough, Wyeth, Bristol Meyers Squibb and Roche, and has received lecture fees from Abbott Laboratories, Wyeth, Roche, Bristol Meyers Squibb and ScheringPlough. LC has received lecture fees from Abbott, Schering-Plough and Roche.

Ethics approval Ethical approval for this study was obtained from the Institutional Review Board (IRB) of the Hospital Ramón y Cajal, Madrid.

Provenance and peer review Not commissioned; externally peer reviewed.

Contributors IGD: interpretation, writing; BPZ: data management, interpretation, writing; MAD: data management, analysis; RR: data collection, verification, interpretation; MVH: data collection, verification, interpretation; JJGR: study design, interpretation, writing; LC: study design, analysis, interpretation, writing.

\section{REFERENCES}

1. Wung PK, Holbrook JT, Hoffman GS, et al. Herpes zoster in immunocompromised patients: incidence, timing, and risk factors. Am J Med 2005;118:1416.

2. Opstelten W, Mauritz JW, de Wit NJ, et al. Herpes zoster and postherpetic neuralgia: incidence and risk indicators using a general practice research database. Fam Pract 2002;19:471-5.

3. Gómez-Reino JJ, Carmona L, Angel Descalzo M. Risk of tuberculosis in patients treated with tumor necrosis factor antagonists due to incomplete prevention of reactivation of latent infection. Arthritis Rheum 2007; 57:756-61.

4. Ito M, Nakano T, Kamiya T, et al. Effects of tumor necrosis factor alpha on replication of varicella-zoster virus. Antiviral Res 1991;15:183-92.

5. Smitten AL, Choi HK, Hochberg MC, et al. The risk of herpes zoster in patients with rheumatoid arthritis in the United States and the United Kingdom. Arthritis Rheum 2007; 57:1431-8.

6. Wolfe F, Michaud K, Chakravarty EF. Rates and predictors of herpes zoster in patients with rheumatoid arthritis and non-inflammatory musculoskeletal disorders. Rheumatology (Oxford) 2006;45:1370-5.

7. Strangfeld A, Listing J, Herzer P, et al. Risk of herpes zoster in patients with rheumatoid arthritis treated with anti-TNF-alpha agents. JAMA 2009;301:737-44.

8. Carmona L, Gómez-Reino JJ, Rodríguez-Valverde V, et al. Effectiveness of recommendations to prevent reactivation of latent tuberculosis infection in patients treated with tumor necrosis factor antagonists. Arthritis Rheum 2005;52:1766-72.

9. Gómez-Reino JJ, Carmona L, Valverde VR, et al. Treatment of rheumatoid arthritis with tumor necrosis factor inhibitors may predispose to significant increase in tuberculosis risk: a multicenter active-surveillance report. Arthritis Rheum 2003;48:2122-7.

10. MedDRA Maintenance and Support Services Organization. http://www. meddramsso.org. (accessed 26 Mar 2010).

11. Ministerio de Sanidad y Consumo. Encuesta Nacional de Salud 2003. http://www.msc.es/estadEstudios/estadisticas/encuestaNacional/home.htm (accessed 27 Oct 2008).

12. Instituto Nacional de Estadística (INE). http://www.ine.es (accessed 28 Nov 2008).

13. Carmona L, Descalzo MA, Perez-Pampin E, et al. All-cause and cause-specific mortality in rheumatoid arthritis are not greater than expected when treated with tumour necrosis factor antagonists. Ann Rheum Dis 2007;66:880-5.

14. Carmona L, Hernández-García C, Vadillo $C$, et al. Increased risk of tuberculosis in patients with rheumatoid arthritis. J Rheumatol 2003;30:1436-9.

15. Spanish Society of Rheumatology. BIOBADASER: Spanish Registry for Adverse Events of Biological Therapies in Rheumatic Diseases. http://biobadaser.ser.es/ biobadaser/eng/index.html. (accessed 26 Mar 2010).

16. Descalzo MA, Biobadaser Scientific Committee (CC), Biobadaser Study Group (SG). Spanish registry for adverse events of biological therapies in rheumatic diseases (BIOBADASER): State Report on January 26th 2006. Reumatol Clin 2007;3:4-20.

17. Terris DD, Litaker DG, Koroukian SM. Health state information derived from secondary databases is affected by multiple sources of bias. J Clin Epidemiol 2007:60:734-41.

18. Calle JE, Saturno PJ, Parra P, et al. Quality of the information contained in the minimum basic data set: results from an evaluation in eight hospitals. Eur J Epidemiol 2000; 16:1073-80

19. Wharton M. The epidemiology of varicella-zoster virus infections. Infect Dis Clin North Am 1996;10:571-81.

20. Nilsson A, Ortqvist A. Severe varicella pneumonia in adults in Stockholm County 1980-1989. Scand J Infect Dis 1996;28:121-3.

21. de Melker H, Berbers G, Hahné S, et al. The epidemiology of varicella and herpes zoster in The Netherlands: implications for varicella zoster virus vaccination. Vaccine 2006;24:3946-52.

22. Torigo S, Ihara T, Kamiya H. IL-12, IFN-gamma, and TNF-alpha released from mononuclear cells inhibit the spread of varicella-zoster virus at an early stage of varicella. Microbiol Immunol 2000;44:1027-31.

23. Marin M, Güris D, Chaves SS, et al. Prevention of varicella: recommendations of the Advisory Committee on Immunization Practices (ACIP). MMWR Recomm Rep 2007; 56(RR-4):1-40.

24. Oxman MN, Levin MJ, Johnson GR, et al. A vaccine to prevent herpes zoster and postherpetic neuralgia in older adults. N Engl J Med 2005;352:2271-84.

25. Peña-Rey I, Martínez de Aragón MV, Villaverde Hueso A, et al. [Epidemiology of varicella in Spain pre- and post-vaccination periods]. Rev Esp Salud Publica 2009;83:711-24. 